\title{
Clinical algorithms for identification and management of delay in the progression of first and second stage of labour.
}

\author{
Julia Pasquale ${ }^{1}$, Mónica Chamillard ${ }^{2}$, Virginia Diaz ${ }^{1}$, Celina Gialdini ${ }^{2}$, Mercedes Bonet $^{3}$, \\ Olufemi Oladapo ${ }^{3}$, and Edgardo Abalos ${ }^{4}$ \\ ${ }^{1}$ Centro Rosarino de Estudios Perinatales \\ ${ }^{2}$ Affiliation not available \\ ${ }^{3}$ World Health Organization \\ ${ }^{4}$ Centro Rosarino de Estudios Perinatales (CREP)
}

November 12, 2020

\begin{abstract}
Aim: To develop clinical algorithms for the assessment and management of slow progress of labour. Population: Low-risk singleton, term, pregnant women in labour. Setting: Institutional births in low- and middle-income countries. Search Strategy: We systematically reviewed the literature on normal labour progression, and guidance on clinical management of abnormally slow progression from 1 December 2015 to 1 December 2020. Case scenarios: We developed two clinical algorithms: one for abnormally slow labour progression and arrest during first and one for second stage. Conclusions: Identifying abnormal progress of labour is often challenging. These algorithms may help to reduce misdiagnosis.
\end{abstract}

\section{SELMA manuscript - BJOG format}

Clinical algorithms for identification and management of delay in the progression of first and second stage of labour.

J Pasquale, ${ }^{\mathrm{a}} \mathrm{M}$ Chamillard, ${ }^{\mathrm{a}}$ V Diaz, ${ }^{\mathrm{a}} \mathrm{C}$ Gialdini, ${ }^{\mathrm{a}} \mathrm{M}$ Bonet,${ }^{\mathrm{b}}$ Olufemi T Oladapo, ${ }^{\mathrm{b}} \mathrm{E}$ Abalos, ${ }^{\mathrm{a}}$ for the

\section{WHO Intrapartum Care Algorithms Working Group *}

a. Centro Rosarino de Estudios Perinatales (CREP) Rosario, Argentina.

b. UNDP/UNFPA/UNICEF/WHO/World Bank Special Program of Research, Development and Research Training in Human Reproduction (HRP), Department of Sexual and Reproductive Health and Research, World Health Organization, Geneva, Switzerland.

\section{Correspondence:}

E-mail:jpasquale@crep.org.ar

\section{Slow labour progress algorithms}

\section{Abstract}

Aim: To develop clinical algorithms for the assessment and management of slow progress of labour.

Population: Low-risk singleton, term, pregnant women in labour.

Setting : Institutional births in low- and middle-income countries. 
Search Strategy: We systematically reviewed the literature on normal labour progression, and guidance on clinical management of abnormally slow progression from 1 December 2015 to 1 December 2020 from relevant international guidelines, Cochrane reviews and primary research studies in PubMed by searching for international and national guidance documents, electronic databases and published systematic reviews using relevant keywords.

Case scenarios : We developed two clinical algorithms: one for abnormally slow labour progression and arrest during first and one for second stage. The algorithms provide definitions of suspected and confirmed slow progress of labour or arrest, initial assessment and ongoing monitoring, differential diagnosis, and management of the abnormalities, as well as links to other algorithms for labour management.

Conclusions: Identifying abnormal progress of labour is often challenging. These algorithms may help to reduce misdiagnosis and the unnecessary use of interventions to accelerate labour. We hope these algorithms will help health providers identify abnormal labour progress and institute prompt management or referral where needed.

Funding: This work was funded by the Bill \& Melinda Gates Foundation (Grant \#OPP1084318); The United States Agency for International Development (USAID); and the UNDP-UNFPA-UNICEF-WHOWorld Bank Special Program of Research, Development and Research Training in Human Reproduction (HRP), a cosponsored program executed by the World Health Organization (WHO). The funders had no role in design, data collection and analysis, decision to publish, or preparation of the manuscript.

Keywords: labour, active first stage, duration, delay, protracted, dystocia, second stage of labour, abnormal, length, prolonged labour.

Tweetable abstract: Evidence-based clinical algorithms may help or standardize early identification and management of abnormally slow labour progress or arrest.

\section{Introduction}

Spontaneous labour has been divided into phases and stages to facilitate clinical management. However, its actual progression has not yet been standardized for all women. The applicability of time limits in practice has been challenged by variations in definitions of the onset, phases and stages of labour. ${ }^{1}$ Active first stage starts at $5 \mathrm{~cm}$ of cervical dilatation, and for women with spontaneous labour onset, it usually does not extend beyond 12 hours in first labours, and 10 hours in subsequent labours. ${ }^{2-3}$ The second stage of labour duration has been proposed to last up to 2 hours in nulliparous (up to 3 hours if they had regional anaesthesia) and up to 1 hour or 2 hours in parous women without or with regional anaesthesia, respectively. ${ }^{4-5}$

It is difficult to determine the incidence of prolonged labour, because the accepted thresholds of normal and protracted labour ${ }^{6}$ and the diagnostic criteria for dystocia are not yet universally standardized and WHO is intensively working on achieving this. Therefore, prolonged labour reported widely ranges from $1.3 \%$ to $37 \%$ of all deliveries. ${ }^{7-8-9}$

Prolonged first stage of labour may be due to poor uterine activity, fetal size, abnormal fetal position or presentation, pelvic abnormalities resulting in cephalo-pelvic disproportion (CPD), or psychological reasons. ${ }^{10}$ Second stage may get unduly prolonged because of CPD, abnormal fetal position, and poor expulsive efforts resulting from conduction analgesia, sedation or maternal exhaustion. ${ }^{11}$ The consequences of a prolonged first stage and second stage could be postpartum haemorrhage, low 5-minute Apgar score or admission to the neonatal intensive care unit. ${ }^{11}$

Identifying slow progress that justifies interventions to accelerate labour is often challenging in clinical practice. This may have contributed to increasing interventions during labour and childbirth particularly the increased use of oxytocin augmentation and caesarean section for "failure to progress in labour", ${ }^{12-13}$ and associated maternal and newborn morbidities. ${ }^{14-15-16}$ Diagnosis and management of prolonged second stage of labour and its complications often poses a dilemma to the health provider regarding timing and type of 
intervention. ${ }^{11}$ This is the stage in labour where the contribution of a skilled birth attendant is the most critical in ensuring a safe outcome. ${ }^{17}$

The following algorithms aim to guide health care providers in the management of spontaneous labour in women at low risk of perinatal complications and to summarize the clinical pathways for identifying, diagnosing, managing, and monitoring deviations from normal observations of labour and its progress.

\section{Methods}

This work is part of a project commissioned by the WHO, for the management of uneventful and complicated labours, to complement WHO intrapartum care guidelines, and facilitate the development of electronic evidence-based, decision-support tools by stakeholders, to improve labour outcomes in low-resource settings 18

We developed algorithms for two case scenarios, based on findings of a review on slow labour progress (first and second stage), identification of the most common deviations, severity of related complications or clinical outcomes and discussion with the WHO Intrapartum Care Algorithms Working Group. These were as follows: slow progress of first and second stage. We also included fetal abnormal presentation as a contributing factor for progression abnormalities in both scenarios.

A detailed description of the methodology used for the development of these algorithms is provided in elsewhere. ${ }^{19}$

\section{Search strategy}

We developed a systematic approach for literature search to construct the algorithm of labour progression abnormalities.

We searched the following list of databases from 1 December 2015 to 1 December 2020: The Cochrane Library and Cochrane Central Register of Controlled Trials. We searched for existing WHO guidelines and recommendations. We also searched for guidelines from the International Federation of Gynaecology and Obstetrics (FIGO), the National Institute for Health and Care Excellence (NICE), the American College of Obstetricians and Gynaecologists (ACOG) and the Royal College of Obstetricians and Gynaecologists (RCOG). We completed the search in PubMed to identify all other clinical practice guidelines, systematic reviews, meta-analyses, and relevant single studies published in the last 5 years. Searches included (labour AND ("active first stage" OR duration OR delay OR protracted OR dystocia)) ; "second stage of labour" AND ("abnormal" OR "length" OR "prolonged"); and ("Labour dystocia" OR ("Breech presentation" OR "Brow presentation" OR "Face presentation" OR "Transverse presentation" OR "Oblique presentation") OR (labour AND (breech OR transverse OR malposition)) in Title Abstract Keyword, with all possible word variations. The search was limited to English language.

The literature review was guided by the hierarchy of evidence and prioritised WHO guidelines followed by other international or national guideline. In the absence of guidelines on a case scenario, a combination of existing studies and expert opinion was used to determine key points for consideration in the algorithm. The highest level of evidence found was used to support the decisions along the management pathway, in the order of up-to-date systematic review (with meta-analyses), up-to-date systematic review (without meta-analyses), any available systematic review, validated decision rules, randomized controlled trials, nonrandomized controlled trials, observations studies, and consensus documents.

Two reviewers CG-MC independently screened the title and the abstract, extending manual searching through the reference lists of relevant articles and extracted the recommendations and supporting evidences into an excel file. Inconsistencies were resolved by a third reviewer VD.

\section{Population and setting}

The algorithms were developed to cover management of pregnant women with singleton, term pregnancies considered to be at low risk of developing complications at admission to the birthing facility, with the 
diagnosis of spontaneous onset of labour. Health facilities in low- and middle-income countries (LMIC) were the priority. However, the algorithms are applicable to any health care setting, and possible adaptations that may be required were acknowledged. The target users for these algorithms are skilled health personnel providing care during childbirth working alone or as part of teams, particularly midwives, non-specialized clinicians (i.e. clinicians without specialist training in obstetrics but who also provide care for women in labour), and specialists.

\section{Development of algorithm}

After collating the evidence, a selection process for inclusion of the evidence in the algorithm took place. Selection was based on relevance of the evidence to the key decision points and severity of the condition targeted by an intervention. The selection also accounted for the strength of evidence and applicability and feasibility in a LMIC context. If there were inconsistencies among guidelines, the most up-to-date guideline and evidence were reviewed and used to inform the algorithms in consultation with the WHO Intrapartum Care Algorithms Working Group. Algorithms were structured to cover criteria that should be used to suspect a deviation from normal parameters in labour, initial assessment, probable causes and potential differential diagnosis, further assessments to reach the most probable diagnosis, and management of the condition. Draw.io, an open source diagramming online software was used to construct the algorithm in a flowchart format. The algorithm was composed of standardised but variable shaped boxes, representing either a clinical state (rounded rectangle), decision point (diamond or hexagon), action task (rectangle), or link (oval) to a different algorithm. Each box was numbered and joined to other boxes via arrows, to orientate the reader to the direction of flow. The numbers also corresponded to a table of evidence, showing the evidence source for the action and decision points. The algorithms underwent internal peer review by the WHO Intrapartum Care Algorithms Working Group.

\section{Results}

\section{Search results}

A flow diagram describing the search results is shown in Figure S1. Initial search retrieved 47 potentially relevant clinical practice guidelines, 471 Cochrane systematic reviews, and 8871 primary papers. From these, 10 clinical practice guidelines, three Cochrane systematic reviews and 16 original papers were finally selected.

\section{-Case scenarios for labour progression abnormalities}

We considered two case scenarios for labour progress abnormalities: slow progress of active first stage and slow progress of second stage of labour.

\section{General Considerations:}

All women with an uncomplicated term pregnancy admitted during active first or second stage should be evaluated by a skilled birth attendant. The contribution of a qualified and skilled attendant with midwifery skills is critical for ensuring good outcome. ${ }^{17-20}$ All women should receive emotional support through labour companionship and continuous support during labour and delivery. They should also be encouraged to adopt a birth position of their choice (including upright positions). Women and companions must be informed about the progress of labour, possible reasons for delays explained and care options effectively and respectfully communicated. ${ }^{2}$ If a deviation from the adequate progress of any stage of labour occurs, it is mandatory to call for a second person trained to assist and consider the transfer to an obstetric-led care unit. ${ }^{17-21}$

Maternal clinical assessments (maternal pulse, blood pressure, temperature) as well as the fetal assessments have to be performed frequently. Health care providers should palpate the woman's abdomen for a prompt and thorough clinical assessment to rule out a full bladder and encourage the woman to empty her bladder regularly. Leopold's manoeuvres are the standard method to determine fetal size, fetal presentation, position, engagement of the presenting part and descent. By digital vaginal examination, cervical dilatation, fetal presentation, position and attitude should be evaluated in all women admitted in labour. ${ }^{2-22-23-24}$ 
If breech presentation is identified, the health care provider should offer a caesarean section, where possible and appropriate. If abdominal delivery is not possible or consented to, breech vaginal birth should be assisted by a well-trained skilled birth attendant. Women and health care providers should be aware of its potential complications. ${ }^{21}$

Fetal wellbeing should be assessed during the entire process of labour and birth. In low risk women, the recommended method for fetal heart auscultation is intermittent fetal heart rate auscultation using Doppler ultrasound or Pinard fetal stethoscope. Evidence shows that continuous cardiotocography (CTG) on admission in labour probably increases the risk of caesarean section without improving birth outcomes as well as the likelihood of a woman and her baby receiving a cascade of other interventions. ${ }^{3}$ Diagnosis and management of fetal heart rate abnormalities are covered in another manuscript in this series. ${ }^{25}$

\section{Scenario 1. Slow progression of active first stage of labour (Figure 1)}

\section{Condition}

The active first stage is a period of time characterized by regular painful uterine contractions, a substantial degree of cervical effacement and more rapid cervical dilatation from $5 \mathrm{~cm}$ until full dilatation for first and subsequent labours. ${ }^{2-3-5}$ It is fundamental to reaffirm and reassess that the woman is in active first stage

The median duration of active first stage is 4 hours $\left(95^{\text {th }}\right.$ centile is up to 12 hours) in first labour and 3 hours $\left(95^{\text {th }}\right.$ centile is up to 10 hours) in second and subsequent labours, when the starting point of reference is $5 \mathrm{~cm}$ cervical dilatation. A cervical dilatation rate of less than $0.5 \mathrm{~cm}$ to $1 \mathrm{~cm}$ per hour during this phase is commonly considered to be slow progress of labour, with the trigger for intervention stipulated at a variable period following such deviation. While it is widely acknowledged that labour tends to be faster among multiparous compared to nulliparous women, the same criteria often apply for identification of delay in both groups in clinical practice. ${ }^{2-3-5}$

\section{Assessment}

Health care providers should assess maternal general conditions (pulse, blood pressure, temperature, urine output). They should also evaluate frequency, intensity and duration of contractions in 10 minutes, cervical dilatation, fetal presentation, position and attitude by digital vaginal examination and Leopold's manoeuvres, in all women admitted in labour. These would help to determine the possibility of giving birth vaginally because abnormal fetal presentations and attitude are causes of labour dystocia. ${ }^{2-22-23-24}$ By offering vaginal examination, health providers will also be able to assess asses state of membranes, liquour characteristics, presence of bleeding or discharge and cervical effacement. If the following signs are present: presenting part outside the pelvis, ballooning of lower uterine segment, formation of retraction band or maternal and fetal distress, suspect obstructed labour or cephalopelvic disproportion. ${ }^{4}$

There are interventions that have been proved to prevent delay in progress of active phase of first stage: the continuous support during labour, labour companionship, the use of comfortable position of woman's choice, and free mobility. ${ }^{2}$ To provide adequate pain relief, available options must be discussed with the woman prior to the onset of labour and offered according to her wishes and using health facility protocols and norms. For women at low risk, oral fluid and food intake during labour is recommended. ${ }^{2}$

\section{Treatment}

If slow progression of active first stage of labour is associated with lack of regular uterine contractions, the stimulation of uterine contractions with oxytocin and amniotomy is a reasonable clinical choice. There is lack of evidence on how the sequence of amniotomy and oxytocin infusion affects outcomes and this is a research priority. ${ }^{26}$ Augmentation of labour is the process of stimulating the uterus to increase the frequency, duration and intensity of contractions after the onset of spontaneous labour. It has commonly been used to treat delayed labour when uterine contractions are assessed to be insufficiently strong or inappropriately coordinated to dilate the cervix. Labour augmentation has traditionally been performed with the use of intravenous oxytocin infusion and/or artificial rupture of amniotic membranes (amniotomy). ${ }^{26}$ Augmentation 
with oxytocin alone should be performed when indicated as treatment of confirmed delay in progress of labour. For a multiparous woman with confirmed delay in the established first stage of labour, an obstetrician should perform a full assessment, including abdominal palpation and vaginal examination, before a decision is made about using oxytocin. If oxytocin is used, the time between increments of the dose is no more frequent than every 30 minutes until there are $4-5$ contractions in 10 minutes. The start dose of oxytocin for augmentation, and the increments, should be the subject of further research. ${ }^{10}$

The decision to rupture the membranes should be made on the basis of other clinical considerations of the progress of labour, after explaining to the woman and her companion what the procedure involves and that it will shorten labour by about an hour and may increase the strength and pain of contractions. It is also mandatory to have special consideration if the woman has HIV or HBV, where amniotomy is best avoided to reduce risk of infection to the baby. Whether or not a woman has consented to an amniotomy, all women with suspected slow progression of active first stage should be advised to have a vaginal examination 2 hours later, and a diagnosis of delay is made if progress is less than $1 \mathrm{~cm} .{ }^{10}$ If thick meconium is evident or fetal distress is suspected through abnormal fetal heart rate auscultation, it is necessary to have a medical review for a plan of care when there are multiple co-existent problems to decide the mode of birth. Management of uterine contraction abnormalities meconium stained amniotic fluid are addressed in other manuscripts in this series. ${ }^{27}$

Where obstructed labour or cephalopelvic disproportion is suspected, a medical review should be undertaken to consider delivery by $\mathrm{C}$-section and preparations should be made for neonatal resuscitation. ${ }^{4}$

\section{Scenario 2. Slow progress of second stage of labour (Figure 2)}

\section{Condition}

The second stage of labour is the period of time between full cervical dilatation and birth of the baby, during which the woman has an involuntary urge to bear down, as a result of expulsive uterine contractions. ${ }^{2}$

Slow progress of second stage of labour can be suspected when birth does not take place within 2 hours (or 3 hours if regional anaesthesia is used) for nulliparous women since the start of the active phase and within 1 hour (or 2 hours if regional anaesthesia is used) for parous women. ${ }^{4-5}$

Assessment

Slow progress of second stage requires clinical reassessment of the women and the fetus. Assessment should be made of fetal size, adequacy of the pelvis, fetal wellbeing, and maternal pushing efforts. The abdomen should be palpated for a prompt and thorough clinical assessment to rule out full bladder, and abdominal examination may reduce the number of vaginal exams that are necessary. Vaginal examination should be offered to evaluate the state of the membranes and amniotic fluid characteristics, bleeding, discharge, effacement of the cervix, fetal presenting part, position and descent or the presence of caput or moulding. If characteristics such as large caput, third degree moulding, cervix poorly applied to presenting part, oedematous cervix, ballooning of lower uterine segment, formation of retraction band, or maternal or fetal distress are present, obstructed labour or cephalopelvic disproportion should be suspected.

\section{Treatment}

Efforts should be made to achieve vaginal birth where appropriate. Amniotomy should be offered if the membranes are intact. Adequate pain relief should be provided, available options must be discussed with the woman prior to the onset of labour and offered according to her wishes and using health facility protocols and norms.

Operative interventions are unlikely to be required when maternal and fetal conditions are satisfactory and there is evidence of descent of the presenting part. On the other hand, if thick meconium is evident or fetal distress is suspected, it is necessary to have a medical review for a plan of care in the presence of multiple abnormalities, to decide the mode of birth. Management of thick meconium-stained amniotic fluid is described in another manuscript in this series. ${ }^{27}$ If obstructed labour or cephalopelvic disproportion is 
suspected, a medical review is needed to consider delivery by C-section and preparations should be made for neonatal resuscitation.

Safe assisted vaginal birth requires a careful assessment of the clinical situation, clear communication with the woman and healthcare providers, and expertise in the chosen procedure. The health provider should choose the instrument most appropriate to the clinical circumstances and their level of skill and they should be aware that the use of forceps or vacuum extraction have different benefits and risks. Failure to complete the birth with a single instrument is more likely with vacuum extraction, but maternal perineal trauma is more likely with forceps. Soft cup vacuum extractors have a higher rate of failure but a lower incidence of neonatal scalp trauma. ${ }^{28}$ Instrumental delivery should only be attempted by care providers who are trained and qualified to recognize the indications, and are skilled and equipped to perform the procedure safely for mother and baby. ${ }^{17}$ More information on indications, conditions to perform, precautions and supervision of assisted delivery can be found in a recent published guideline. ${ }^{28}$

\section{Discussion}

\section{Main findings}

We have developed evidence-based algorithms for intrapartum assessment and provide guidance on management of common labour progress abnormalities, namely labour arrest and slow progress of $2^{\text {nd }}$ stage. The algorithms were developed for all settings, but with a focus in places with limited resources.

\section{Strengths and limitations}

The algorithms have been developed by using best available, up to date evidence with a systematic approach for gathering the evidence-based and develop the pathways. Particularly for labour arrest, the evidence is scarce. Links with other evidence-based algorithms are provided where relevant and escalation for specialist care is highlighted where needed. However, given the lack of evidence for some critical points in the pathways we had to rely on expert opinion. The algorithms focus only on delay in progress, but they do not cover management of labour in high-risk women or those who may present with multiple complications. Implementation of the algorithms may be limited by the availability of resources, such as cardiotocography.

\section{Interpretation}

Identifying abnormal progress of labour that justifies a medical intervention is often challenging. Thus, 'failure of labour to progress' -a term poorly defined but generally accepted -has become a leading indication for oxytocin augmentation and primary caesarean section. ${ }^{3}$ The implementation of the 5 -cm cervical dilatation as benchmark of the active phase of the first stage of labour might be cost-effective because it reduces the use of interventions such as caesarean section, oxytocin augmentation, cardiotocography, pain relief and antibiotics. $^{2}$ It is expected that the algorithms on labour arrest and delay in second stage of labour presented here, will help healthcare providers to follow a more conscious management of these conditions, respecting labour progress and avoiding unnecessary interventions.

These algorithms should be adapted to local context, taking into considerations availability of skilled personnel and other resources.

Developing the algorithm highlighted the need for more data on the epidemiology of delay in the progression of first and second stage of labour and its management in the light of the new definitions of active first stage and second stage in recently published WHO recommendations. ${ }^{2}$

\section{Conclusions}

\section{Implications for clinical practice}

We have developed clinical algorithms that may help guide decision making with respectful care during delays identified during the first and second stages of labour. The use of these algorithms may help to reduce the misdiagnosis of dystocia in early labour, and could potentially reduce the unnecessary use of interventions to accelerate labour. 


\section{Implications for research}

This pathway is likely to be applied and evaluated in different settings and in different countries, whose feedback on implementation and outcomes should be incorporated in order to improve its external validity and, therefore, the quality of care. Clinicians, researchers and policy makers should be encouraged to adapt these algorithms in accordance with their own context, considering the local availability of resources, the acceptability of key stakeholders, centred in respectful maternity care dimensions and equity principles.

\section{Author contributions}

MB and OTO conceived methodology for the development of intrapartum care algorithms for management of labour. CG performed the screening of search outputs, identified eligible studies, and extracted data. MC performed the data analysis and developed the algorithms with inputs from CG, VD, JP and the WHO Intrapartum Care Algorithms Working Group. JP wrote the first draft of the paper. All authors revised and approved the final version of the manuscript.

\section{Disclosure of interest}

EA, MB, OTO, MC, VD, CG and JP have no conflicts of interest to declare.

\section{Funding}

This work was funded by the Bill \& Melinda Gates Foundation (Grant \#OPP1084318); The United States Agency for International Development (USAID); and the UNDP-UNFPA-UNICEF-WHO-World Bank Special Programme of Research, Development and Research Training in Human Reproduction (HRP), a cosponsored program executed by the World Health Organization (WHO). The funders had no role in design, data collection and analysis, decision to publish, or preparation of the manuscript.

\section{WHO Intrapartum Care Algorithms Working Group}

(In alphabetical order) Livia Ciabati, Lariza Laura De Oliveira (University of São Paulo, Brazil); Joyce Browne, Marcus Rijken (UMC Utrecht, the Netherlands); Sue Fawcus (University of Cape Town, South Africa); Justus Hofmeyr (Frere Maternity Hospital East London, South Africa); Tippawan Liabsuetrakul (Prince of Songkla University, Thailand); Çağri GÜLÜMSER (University of Health Sciences, Turkey); Anna Blennerhassett (University of Birmingham, United Kingdom), David Lissauer (University of Liverpool, United Kingdom), Shireen Meher (Birmingham Women's and Children's NHS Foundation Trust). World Health Organization Secretariat (Switzerland): Fernando Althabe, Mercedes Bonet, A. Metin Gülmezoglu, Olufemi Oladapo

\section{References}

1. Hanley GE, Munro S, Greyson D, Gross MM, Hundley V, Spiby H, et al. Diagnosing onset of labor: a systematic review of definitions in the research literature. BMC Pregnancy Childbirth 2016; 16:71.

2. WHO recommendations: intrapartum care for a positive childbirth experience. Geneva: World Health Organization; 2018. Licence: CC BY-NC-SA 3.0 IGO.

3. Oladapo OT, Diaz V, Bonet M, Abalos E, Thwin SS, Souza H, et al. Cervical dilatation patterns of "lowrisk" women with spontaneous labour and normal perinatal outcomes: a systematic review. BJOG. 2017. doi:10.1111/1471-0528.14930.

4. NICE Pathway last updated: 29 November 2019. Delay and complications in second stage of Labour. http://pathways.nice.org.uk/pathways/intrapartum-care

5. Abalos E, Oladapo OT, Chamillard M, Díaz V, Pasquale J, Bonet M, et al. Duration of spontaneous labour in "low-risk" women with "normal" perinatal outcomes: a systematic review. Eur J Obstet Gynecol Reprod Biol. 2018;2 23:123-32.

6. Abalos E, Chamillard M, Díaz V, Pasquale J, Souza JP. Progression of the first stage of spontaneous labour [published online ahead of print, 2020 Mar 7]. Best Pract Res Clin Obstet Gynaecol. 2020;S15216934(20)30038-9. doi:10.1016/j.bpobgyn.2020.03.001 
7. Sheiner E, Levy A, Feinstein U, Hallak M, Mazor M. Risk factors and outcome of failure to progress during the first stage of labor: a population-based study. Acta Obstet Gynecol Scand. 2002;81(3):222226.

8. Nystedt A, Hildingsson I. Diverse definitions of prolonged labour and its consequences with sometimes subsequent inappropriate treatment. BMC Pregnancy Childbirth. 2014;14:233. Published 2014 Jul 16. doi:10.1186/1471-2393-14-233

9. Kjaergaard H, Olsen J, Ottesen B, Dykes AK. Incidence and outcomes of dystocia in the active phase of labor in term nulliparous women with spontaneous labor onset. Acta Obstet Gynecol Scand. 2009;88(4):402-407. doi:10.1080/00016340902811001

10. NICE guidelines Intrapartum care: Care of healthy women and their babies during childbirth December 2014. Updated February 2017.

11. Singh S, Kohli U, Vardhan S. Management of prolonged second stage of labor. International Journal of Reproduction, Contraception, Obstetrics and Gynecology 2018, 7 (7), 2527-2531. doi:http://dx.doi.org/10.18203/2320-1770.ijrcog20182855

12. Betran AP, Ye J, Moller AB, Zhang J, Gülmezoglu AM, Torloni MR. The increasing trend in caesarean section rates: global, regional and national estimates: 1990e2014. PloS One 2016;11:e0148343.

13. WHO Reproductive Health Library. WHO recommendation on respectful maternity care. (February 2018). The WHO Reproductive Health Library; Geneva: World Health Organization.

14. Keag OE, Norman JE, Stock SJ. Long-term risks and benefits associated with cesarean delivery for mother, baby, and subsequent pregnancies: Systematic review and meta-analysis. PLoS Med. 2018;15(1):e1002494.

15. Timor-Tritsch IE, Monteagudo A. Unforeseen consequences of the increasing rate of cesarean deliveries: early placenta accreta and cesarean scar pregnancy. A review. Am J Obstet Gynecol. 2012;207(1):14-29.

16. Cook JR, Knight M, Dhanjal MK. Multiple repeat caesarean section in the UK: incidence and consequences to mother and child. A national, prospective cohort study-authors' reply. BJOG. 2013;120(9):1155.

17. FIGO GUIDELINES Management of the second stage of labor 2012.https://obgyn.onlinelibrary.wiley.com/doi/full/10.1016/j.ijgo.2012.08.002

18. Souza JP, Oladapo OT, Bohren MA, et al. (2015). The development of a Simplified, Effective, Labour Monitoring-to-Action (SELMA) tool for Better Outcomes in Labour Difficulty (BOLD): study protocol. Reprod Health. 2015 May , 26;12:49. doi: 10.1186/s12978-015-0029-4.

19. Bonet M, Oladapo OT. Development of evidence-based intrapartum care algorithms: methodological considerations. BJOG.

20. Perinatal Practice Guideline. South Australian Perinatal Practice Guidelines. April 2017

21. NICE 2019. Intrapartum care for women with existing medical conditions or obstetric complications and their babies. NG 121. ISBN: 978-1-4731-3296-2.

22. D. M. Sherer, M. Miodovnik, K. S. Bradley and O. Langer. Fetalhead position during the second stage of labor: comparison of digital vaginal examination and transabdominal ultrasonographic examination. Ultrasound Obstet Gynecol 2002; 19: $264-268$

23. Breech Presentation. Medscape.

24. Hofmeyr GJ, Kulier R, West HM. Expedited versus conservative approaches for vaginal delivery in breech presentation.Cochrane Database of Systematic Reviews 2015, Issue 7. Art. No.: CD000082. DOI: 10.1002/14651858.CD000082.pub3.

25. Hofmeyr J. Clinical decision pathways to identify causes and management options for fetal heart rate abnormalities.

26. WHO recommendations for augmentation of labour. Geneva: World Health Organization; 2014.

27. Gulumser C, Yassa M. Clinical management of uterine contraction abnormalities: an evidence-based intrapartum care algorithm.

28. Murphy, DJ, Strachan, BK, Bahl, R, on behalf of the Royal College of Obstetricians Gynaecologists. Assisted Vaginal Birth. BJOG 2020; 127: e70- e112.

\section{Hosted file}


Table S1 S2 Slow progress 1st stage.pdf available at https://authorea.com/users/375220/ articles/492533-clinical-algorithms-for-identification-and-management-of-delay-in-theprogression-of-first-and-second-stage-of-labour

\section{Hosted file}

Table S1 S2 Slow progress 2nd stage.pdf available at https://authorea.com/users/375220/ articles/492533-clinical-algorithms-for-identification-and-management-of-delay-in-theprogression-of-first-and-second-stage-of-labour

\section{Hosted file}

Labour algorithms.pdf available at https://authorea.com/users/375220/articles/492533clinical-algorithms-for-identification-and-management-of-delay-in-the-progression-offirst-and-second-stage-of-labour

\section{Hosted file}

Figure S1. Search results flowchart.pdf available at https://authorea.com/users/375220/ articles/492533-clinical-algorithms-for-identification-and-management-of-delay-in-theprogression-of-first-and-second-stage-of-labour 